haltens wirkt dadurch wenig überzeugend. Wenn man schon die nationalen Mittelwerte der Wahlkreisgrößen benutzt, müsste auch die Parteienfragmentierung durch den Durchschnitt der Fragmentierung in den Wahlkreisen gemessen werden. Da dieses Kapitel für den Hauptargumentationsgang aber nebensächlich ist, wird die eigentliche Leistung der Arbeit nicht geschmälert. Diese besteht in der konzisen Analyse der Mikrozusammenhänge und der überzeugenden Verknüpfung zweier bislang viel zu isolierter Forschungsgebiete.

Insgesamt legt Aiko Wagner eine sehr gelungene Studie vor, die der Wissenschaft noch lange Zeit Inspiration für weitere Projekte liefern wird. Dem Autor kann nur empfohlen werden, seine Arbeit ins Englische übersetzen zu lassen, um die Erkenntnisse auch der internationalen Politikwissenschaft zugänglich zu machen.

Philipp Weinmann

\title{
Die Linke in Deutschland: facettenreiches Porträt, lohnende Lektüre
}

Oppelland, Torsten und Hendrik Träger: Die Linke. Willensbildung in einer ideologisch zerstrittenen Partei (Reihe "Die politischen Parteien der Bundesrepublik Deutschland“), Nomos Verlagsgesellschaft, Baden-Baden 2014, 263 Seiten, € 19,90.

In Zeiten des entideologisierten, unterschiedsarmen Pragmatismus hat ein Buch über die Linke als letzter „ideologischer“ Partei seinen besonderen Reiz. Was indes solch eine Partei dafür bezahlt, nämlich ideologische Zerstrittenheit, wird von Torsten Oppelland und Hendrik Träger als Untertitel ihres Buches gleich mitgeliefert.

Der einleitenden typologischen Charakteristik und Einordnung Der Linken folgt die Darstellung ihrer historischen und programmatischen Entwicklung, sodann die der inneren Willensbildungsprozesse und endet schließlich bei ihrem Wählerpotenzial und ihren strategischen Optionen. Das Besondere der Linken machen die beiden Autoren am typologischen Profil der Partei fest. Sie bildet einen Hybrid, der aus einer Mixtur aus Weltanschauungs-, Sammlungs-, Klassen- und Regionalpartei konstituiert wird. Dass gerade auf diese hoch interessante Kombination von Parteitypen, denen später sogar noch die Volkspartei beigefügt wird, ohne gründlichere Herleitung zurückgegriffen wird, könnte von dem einen oder anderen Kenner der typologischen Parteienforschung moniert werden.

Die Leitfrage lautet, „wie Die Linke mit diesem diffusen Charakter, mit ihrer großen innerparteilichen Heterogenität und den verschiedenen historisch-kulturellen Traditionen umgeht und wie die innerparteiliche Willensbildung in einer solchen Partei funktioniert" (S. 16).

Bei der Darstellung der historischen und programmatischen Entwicklung Der Linken gehen Oppelland und Träger bis zur 1914 beginnenden Abspaltung der USPD von der SPD und der 1919 gegründeten KPD zurück, um dieses Schisma in eine Linie mit der Abspaltung der WASG im Gefolge der Schröderschen Agenda-Reformen von 2003 zu stellen. Auch bei den Ausführungen zur SED geht es ihnen in erster Linie um das Verhältnis zur SPD, um einen Bogen zur Linken zu schlagen - ziemlich viel SPD, wo es doch um eine Genealogie der PDS/Die Linke geht und auch darum, wie Die Linke Vergangenheitsbewäl- 
tigung betreibt. Zum Kern der Sache kommt das Buch ab dem Kapitel „Von der SED zur PDS“ (S. 43 ff.), das eindringlich Prozess und Hintergründe der organisatorischen Kontinuität und ideologischen Abrechnung der PDS mit der SED aufzeigt. Erhellend ist auch anschließend zu erfahren, wie es der PDS gelingt, den Systemzusammenbruch der DDR und die deutsche Einheit organisatorisch und elektoral als „ostdeutsche Regional-und Protestpartei“ (S. 53) zu überleben. Dabei ist sie so breit und schichtenübergreifend in der Wählerschaft aufgestellt, dass ihr in dieser Hinsicht Volkspartei-Charakter zukommt (S. 189 f.).

Ihre Positionierung als linke ostdeutsche Interessenpartei trug indes, wie aufgezeigt wird, zunächst nur in den neuen Bundesländern, nicht jedoch im Westen Früchte. Erst die Fusion der PDS mit der WASG 2007 zur Partei Die Linke brachte einen gesamtdeutschen Durchbruch. Nach dem Desaster der Partei bei der Bundestagswahl 2002 fasste sie in Opposition zur Agenda-Politik von Gerhard Schröder wieder Fuß, was ihr den Wiederaufstieg ermöglichte. Zwar sackte sie bei der jüngsten Bundestagswahl 2013 auf 8,6 Prozent (-3,3) wieder ab, konnte sich damit aber als gesamtdeutsche Linksalternative gegenüber der SPD elektoral konsolidieren. Regierungsbeteiligungen mit der SPD im Osten gingen indes durch die Bank mit „Entzauberungs“-Effekten einher und ließen ihre Wählerbasis schrumpfen.

Die Darstellung der innerparteilichen Willensbildung in der Linkspartei, aufgeschlüsselt nach Personal-, programmatischen Richtungs- und strategischen Entscheidungen, zählt zu den interessantesten Teilen des Buches. Informelle Prozesse entlang der charakteristischen Strömungsgliederung der Partei (S. 111 ff.) geben dabei den Ausschlag. Organisatorisch verfestigte ideologische Strömungen stellen „informelle Machtfaktoren“ dar, die sie als „sozialistische Sammlungs- und Strömungspartei“ (S. 111) kennzeichnen. Zwischen Reformern, Orthodoxen und emanzipatorischer Linken wird der Konflikt um den antikapitalistischen beziehungsweise reformerischen Kurs und die Regierungsbeteiligung ausgetragen. Gestritten wird über „die Rolle der Partei im bestehenden kapitalistischen System“ (S. 130), ohne als „linkspluralistische Sammlungspartei“ (S. 153) eine der Strömungen aus der Partei hinaus zu drängen. Im Personaltableau der Parteispitze (S. 87 ff.) spiegeln sich der ideologische Grabenkampf, aber auch der Strömungsausgleich wider, um eine Spaltung der Partei zu verhindern. Innerparteiliche Willensbildung folgt im Modus entweder dem sanften Druck der Parteiführung, oder die Parteiflügel suchen verhandelnd nach Kompromissen. Dass es auf Parteitagen zum offenen Strömungskonflikt zwischen den Lagern kommt, ist auch möglich.

Wieso es Die Linke mit der ihr innewohnenden Widersprüchlichkeit „noch immer gibt“ (S. 161), erklären Oppelland und Träger mit der Fähigkeit der Partei zum flügelübergreifenden Kompromiss und der Führung durch Vermittlung. Ein Fundus an „gemeinsamen weltanschaulichen Grundüberzeugungen und Zielen“ halte die Partei bei allem Streit über den richtigen Weg zudem zusammen. Eine „Kultur des Misstrauens, der Verdächtigungen“, die sich zwischen den Flügeln eingenistet hat, steht dem aber entgegen.

Ein Hindernis für eine Machtperspektive der Linkspartei an der Seite der SPD ist, dass es ihr seit 2005 nicht nur im Osten im erheblichen Maße gelang, den Sozialdemokraten Arbeiter- und gewerkschaftlich gebundene Wähler abspenstig zu machen. Solange Die Linke an ihrer Konfrontationsstrategie gegenüber der SPD festhält und das Wildern in deren Stammwählerschaft nicht unterlässt (S. 209), bestehen trübe Aussichten für ein rot-rotgrünes Bündnis 2017. 
Das Buch liefert ein facettenreiches, abgewogenes, gelungenes Portrait der Linken, dessen Lektüre auf jeden Fall lohnt. Auch mit seinem umfangreichen statistischen Material zur Wähler- und Mitgliedschaft der PDS/Linke hat es als Nachschlagwerk seinen Wert. Zugleich werden interne Dokumente präsentiert, die Einblicke in die ideologische Selbstbeschreibung und in Richtungskonflikte geben. Mit über 20 Seiten Literaturverzeichnis greift der Band die wesentlichen Befunde und Erkenntnisse des angeschwollenen Literaturbestands über Die Linke auf und liefert der weiteren Forschung, womöglich im Disput, wichtige Anregungen.

Elmar Wiesendahl

\section{Linksextremismus im europäischen Vergleich: methodisch aufwendige, theoretisch zu enge Studie}

Mannewitz, Tom: Linksextremistische Parteien in Europa nach 1990. Ursachen für Wablerfolge und-misserfolge (Reihe Extremismus und Demokratie, Bd. 23), Nomos Verlagsgesellschaft, Baden-Baden 2012, 506 Seiten, $€ 74,-$.

In den letzten 30 Jahren hat sich die vergleichende Wahl- und Parteienforschung intensiv mit den neuen oder erneuerten Parteien am rechten Rand beschäftigt, die vor allem seit den 1980er Jahren in zahlreichen europäischen Ländern entstanden sind. Die Entwicklungen am linken Rand des Parteienspektrums sind darüber oft in Vergessenheit geraten. Tom Mannewitz hat sich in seiner Chemnitzer Dissertation vorgenommen, diese Lücke zumindest teilweise zu schließen, indem er für den Zeitraum seit 1990 die internen und externen Bedingungen für den Erfolg linker Flügelparteien untersucht. Der Epochenbruch von 1990 ist dabei für den Gegenstand in doppelter Weise bedeutsam: Zum einen eröffnet sich für den Parteienvergleich erst durch die Demokratisierung in Mittel- und Osteuropa eine sinnvolle Perspektive. Zum anderen hat der Untergang des Staatssozialismus in Europa auch in den westlichen Ländern die Bedingungen, unter denen Parteien am linken Rand operieren, radikal verändert.

Das Panorama, das Mannewitz entfaltet, ist auf den ersten Blick beeindruckend: Behandelt werden 21 Parteien beziehungsweise Wahlbündnisse in zehn europäischen Ländern (Belgien, Dänemark, Deutschland, Frankreich, Italien, Österreich, Polen, Schweiz, Slowakei und Tschechien). Ob diese Gruppierungen jedoch jeweils in ihrer Gänze als „linksextremistisch“ bezeichnet werden können, erscheint im Falle der deutschen Linkspartei, aber auch der italienischen PRC, der PdCI und des Linksbündnisses „La Sinistra L'Arcobaleno" diskussionswürdig. Dementsprechend vermeidet Mannewwitz zumindest im Fall der Linkspartei in den entsprechenden Textpassagen den Begriff des Extremismus und spricht statt dessen von einer „Linksaußenpartei“, ohne das Problem, dass sich daraus für seine Studie ergibt, zu thematisieren.

Neben Einleitung und Schlussbetrachtung umfasst die Arbeit acht inhaltliche Kapitel. Nach einer konzisen Darstellung der verwendeten Begrifflichkeit und der Kriterien für die Fallauswahl (Kapitel 2) nimmt die fast lehrbuchartige Darstellung der in der Arbeit verwendeten Methode (QCA, Kapitel 3) mit rund fünfzig Seiten ungewöhnlich breiten Raum 